\title{
Contribution of mandibular symphyseal distraction osteogenesis to our therapeutic strategies
}

\author{
L. Pascon ${ }^{1}$, C. Bazert ${ }^{1,2}$, E. Bardinet ${ }^{1,2}$ \\ 1 Specialist certified in dentofacial orthopedics \\ 2 University lecturer, hospital practitioner, Dentistry department, University of Bordeaux 2
}

\begin{abstract}
Distraction osteogenesis helps create new bone by using the natural bone repair process. This technique, applied to the symphysis, affords a new perspective: increasing the mandibular transverse dimension in a stable way due to a simultaneous expansion of the skeletal structure. In this respect, it represents a potential alternative to therapy conventionally implemented to remedy dental crowding and mandibular transverse deficiency. All the same, the technique remains marginal as it is considered to be too invasive and risky. With the exception of the patient's cooperation, the success of mandibular symphyseal distraction osteogenesis (MSDO) relies on three main points: good knowledge of indications, strict compliance with the protocol, and lastly the prevention and management of possible complications. After reviewing these three sections, we will cover a few clinical applications.
\end{abstract}

\section{KEYWORDS}

Midsymphyseal distraction osteogenesis, mandible widening, transverse mandibular deficiency, anterior crowding

\section{INTRODUCTION}

Introduced by Guerrero and his team in the 1990s, mandibular symphyseal distraction osteogenesis (MSDO) has not always been unanimously accepted by orthodontists as it is often deemed to be too invasive or too risky. Nonetheless it still a great asset for the resolution of dental-maxillary disharmony, and it is worthy of its inclusion into our range of therapeutic strategies for treating the mandibular arch.

It is a well-known fact that orthopedic mandibular disjunction is impossible owing to the very premature synostosis of a mandibular symphyseal suture. Orthodontists are therefore faced with two options: they can either raise the first arch via orthodontic expansion
Address for correspondence:

Laurie Pascon - 10 rue Jean-Renaud Dandicolle

33000 Bordeaux

E-mail: laurie.pascon@gmail.com
This is an Open Access article distributed under the terms of the Creative Commons Attribution License (http://creativecommons.org/licenses/by/4.0), which permits unrestricted use, distribution, and reproduction in any medium, provided the original work is properly cited.
Article received: $10-01-2016$ Accepted for publication: 25-2-2016. 
(which can involve a periodontal risk of relapse) or they can create space on the arch by means of stripping, extracting teeth, or even using bone anchorages to lower the lateral sectors.

In this context, mandibular symphyseal distraction offers another perspective: the ability to resolve the lack of space on the inferior arch by simultaneously expanding the dentoalveolar and basal levels. The principles of osteogenic distraction when applied to the symphysis have inspired the work of the Russian orthopedic surgeon llizarov. After an intentional surgical bone fracture, new bone formation must be mechanically stimulated by the progressive traction of the two halves of the mandible. Because of this newly formed bone fabricated via the natural process of bone repair, it is now be possible to increase the mandibular transverse dimension, thus putting an end to the doctrine that the inter-canine length cannot be changed. On a case-by-case basis, this technique offers an alternative to traditional orthodontic solutions, but its success requires that all possible outcomes are considered.

Apart from the cooperation of a patient in following the protocol, the collaboration between an orthodontist and a surgeon is pivotal during the key phases of treatment: from planning the intervention to managing the postsurgical schedule.

This article proposes a summary of all essential theoretical data and practices collected from the literature. The analysis of more than 270 cases contained in these publications has allowed us to pinpoint the complications that constitute a major source of apprehension for practitioners faced with this concept of surgical orthodontics.

\section{INDICATIONS AND CONTRAINDICATIONS FOR SYMPHYSEAL DISTRACTION ATIONS}

\section{Indications}

MSDO is indicated in the following cases:

- In the management of mandibular overcrowding, when conventional methods have reached their limits: Increasing the perimeter of the dental arch is ill-advised because of the delicacy of the periodontium and the strong vestibuloversion of the incisors. Extractions are contraindicated in instances of a flat or concave profile of mandibular relapse after orthodontic treatment with avulsions or of sleep apnea syndrome where the buccal volume is decreased. The specific goal is gaining visible space. If reasonable expansion limits have not been defined, most authors agree to a standard limit of between 6 $\mathrm{mm}$ and $8 \mathrm{~mm}^{15,17,20}$, which equates to half the amount of space gained by extracting the premolars $(15 \mathrm{~mm})$. Distraction osteogenesis should therefore be reserved for cases of moderate-to-severe anterior overcrowding. As with extraction, an orthodontist can appreciate the purpose of expansion; therefore, an orthodontist must account for not only the severity of overlap but also the aims 
of incisor repositioning and the impact of the procedure as it relates to leveling the curve of Spee.

- In the treatment of obstructive sleep apnea hypopnea syndrome (OSAHS) (fig. 1): Malkoç ${ }^{23}$ has shown that symphyseal distraction alone has no significant effect on pharyngeal dimensions. However, when joined to the pharynx, it allows for subsequent expansion. This expansion has beneficial effects on nasal permeability ${ }^{11,28}$, which improves in proportion to the expanded width. Therefore, double expansion, maxillary and mandibular, reduces the apnea-hypopnea index (AHI). In this case, mandibular expansion is guided by the amount of palatal expansion, within the aforementioned limits, to avoid resulting in total maxillary occlusion.

- In the case of true mandibular prognathia, isolated or as part of a syndrome (Treacher Collins, Nagers, etc.): Prognathia can cause Brodie's occlusion that is difficult to treat orthodontically. Conducting MSDO is a possible option to balance the transverse occlusion ${ }^{19}$.

- To achieve alveolar decompensation before mandibular advancement surgery: The space gained from MSDO can be useful for lingually repositioning the mandibular incisors, even more so when there is an associated maxillary expansion (fig. 2).
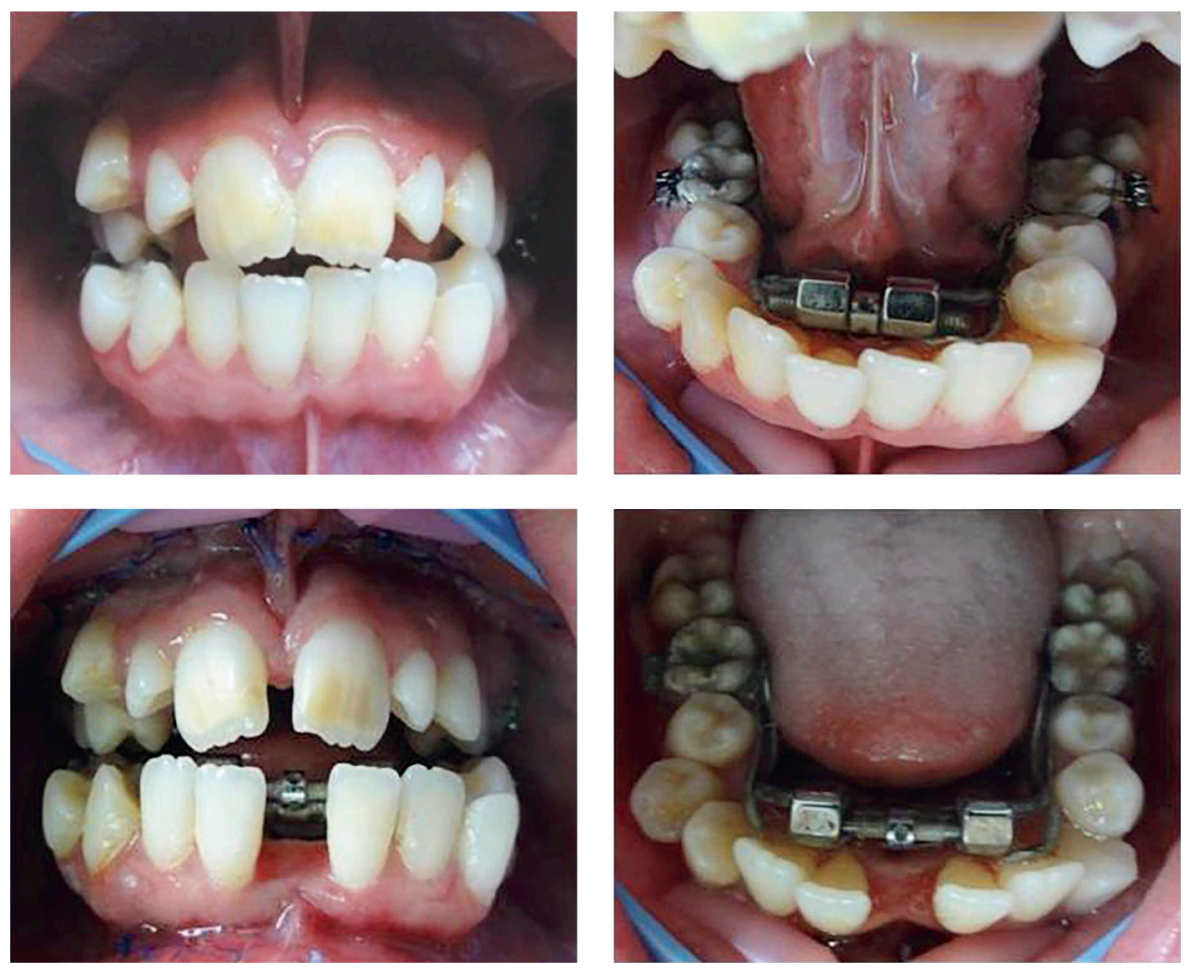

Figure 1

Symphyseal distraction (7 mm) and intermaxillary disjunction in an adolescent patient suffering from OSAHS (AHI of 7) (Dr. El Okeily's case) 
- To facilitate the correction of asymmetric occlusal relationships that originate on the mandible: The space created by MSDO can be used to realign the inferior arch.

- In case of excessive visibility of the buccal corridors when a patient smiles (indication annexed): Significant palatal expansion will improve the esthetics of a smile. Similar to OSAHS, MSDO allows for maxillary procedures.

\section{Contraindications}

Generally, there are no known contraindications specific to symphyseal distraction. It is prohibited when patients are taking intravenous bisphosphonates or were previously exposed to radiotherapy on the orofacial sphere as it can result in osteonecrosis. Locally, there are no absolute contraindications. Nevertheless, the treatment and stabilization of these articular, periodontal, and endodontic conditions are required before performing MSDO.

Finally, age does not seem to be a limiting factor. On the mandible, the trend of cortical apposition and spacing facilitates dentoalveolar expansion during the eruption of permanent teeth. This permits a slight natural increase in the inter-canine distance (Sinclair and Little ${ }^{33}$ ).

Because the symphyseal suture ossifies at around 1 year of age, we cannot expect, as in the maxilla, a transverse growth of the mandibular basal suture even with stimulation. MSDO can therefore be performed on a young patient (if the family deems it necessary) and after being reassured that the osteotomy will not damage any tooth buds. The presence of significant conditions or respiratory distress prompts premature activity elsewhere. In the studies identified, the youngest patients were 7.5 years in Chung's study and 8 years in Ploder's, ${ }^{9,29}$.

\section{KEY STEPS FOR SUCCESSFUL TREATMENT VIA SYMPHYSEAL DISTRACTION}

Treatment via symphyseal distraction proceeds in three steps or stages during which an orthodontist and a surgeon exercise joint control (fig. 2).

\section{The pre-surgical phase: planning the distraction}

Choosing the osteotomy site

To limit the risk of radicular fractures, osteotomy will be performed in an area where the alveolar bone is sufficiently thick. The most suitable site will be jointly determined by a surgeon and $a b$ orthodontist before the intervention, starting with a complete clinical and radiological examination of the canine-incisor zone. A simple, vertical osteotomy between the two central incisors will be prioritized as soon as possible.

In instances of radicular proximity between 31 and 41, it may be worthwhile to consider the following (fig. 3): 


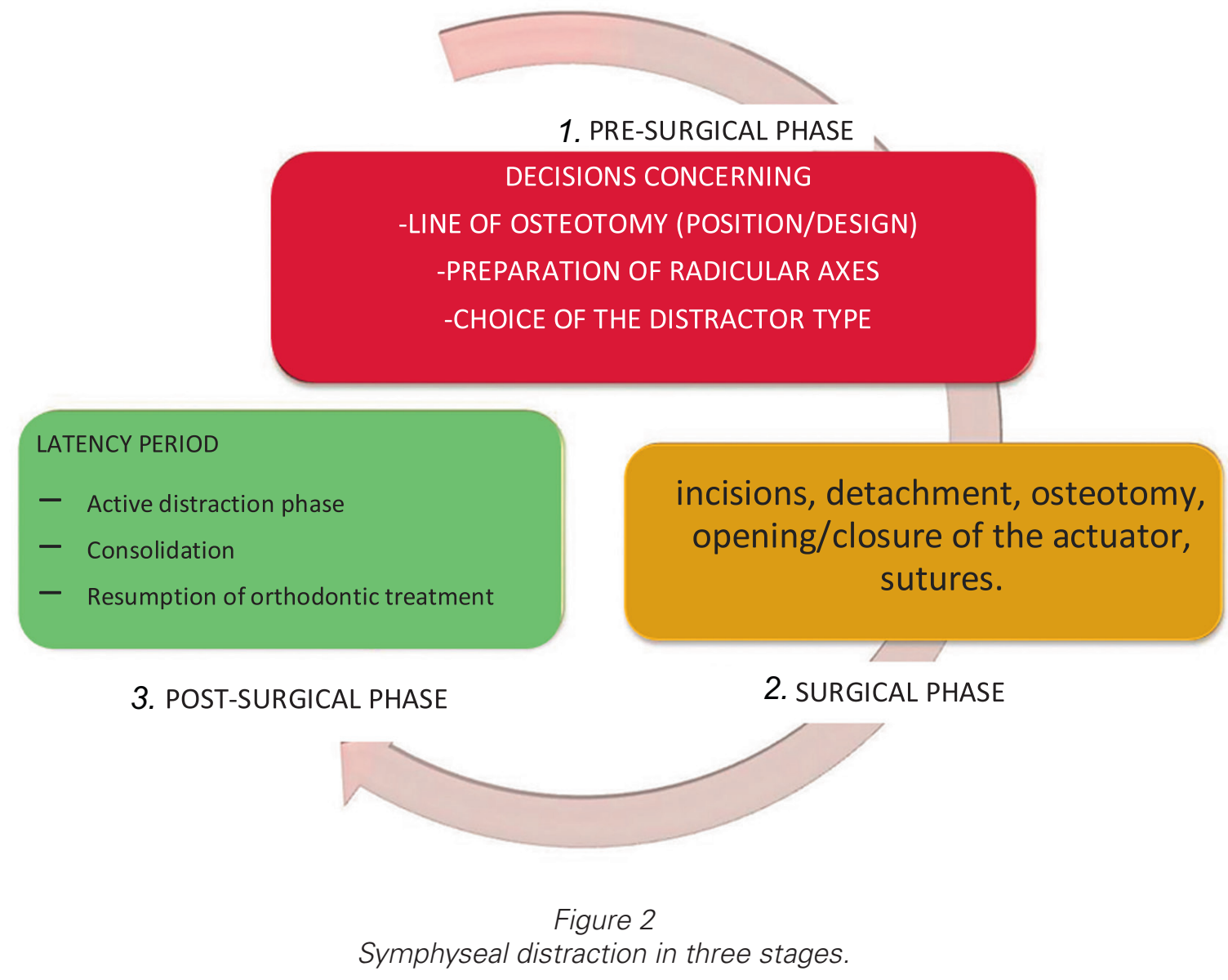

- Shifting the osteotomy line to a parasymphyseal zone or where the thickness of the alveolar bone is sufficient, often between the lateral incisal and the canine zones

- Orthodontically diverging the radicular axes to decrease the risk of fracture or root exposure when making the osteotomy.

\section{Choosing a distractor}

Choosing a distractor can also lead to a discussion between a surgeon and an orthodontist. Dental anchorage tools are attached to the arch via premolar or molar braces. Bone-supported devices and hybrids are directly attached to the symphyseal zone by anchorage plates, with the hybrid device generally supported on the canines (fig. 4).

The criteria for choosing a distractor are identified in the table below (fig. 5).

The rigidity of a distractor is crucial to ensure the stability of the bone callus. It must be able to resist the shear force that can interrupt the vascular network within the regenerate bone, therefore causing an absence of merging or pseudarthrosis (formation of a fibrous callus). However, it should be noted that axial micromovements (compression or tension) will stimulate osteogenisis ${ }^{4}$.

In light of the literature, it would seem that the three types of distractors permit the reliable expansion of 


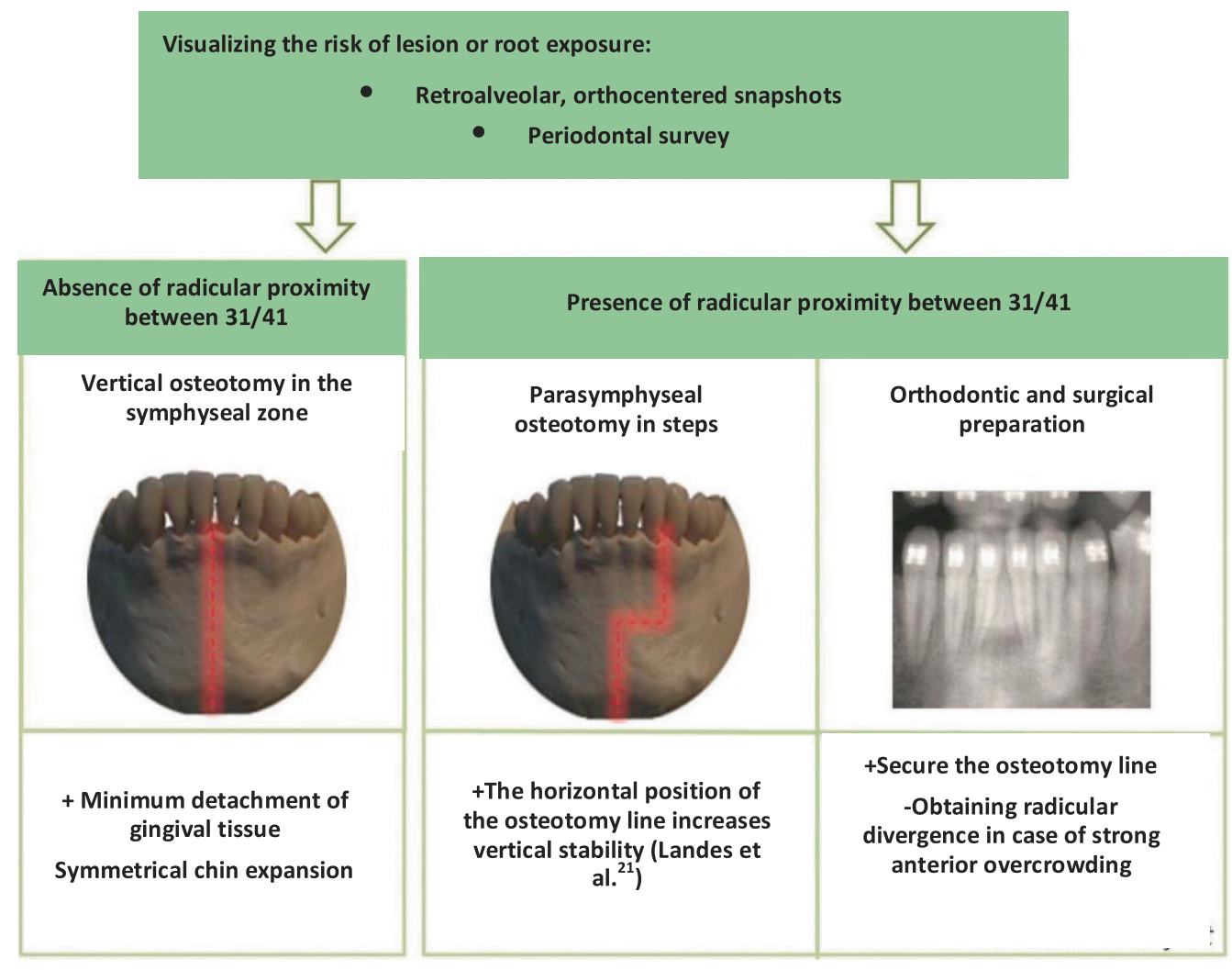

Figure 3

Surgical planning.
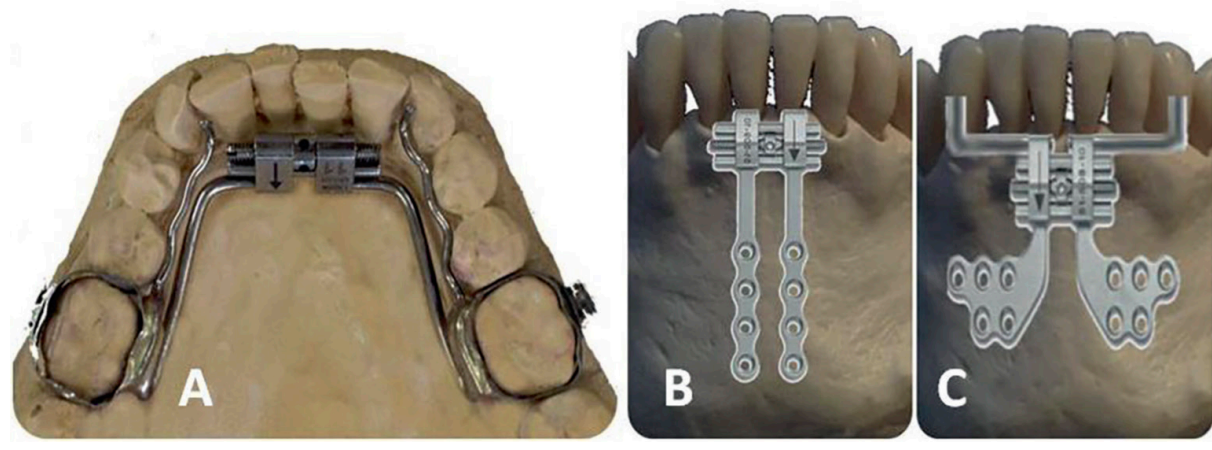

Figure 4

Three types of distractors (A) tooth-supported, (B) bone-supported (Rotterdam Midline Distractor form KLS Martin laboratory), and (C) hybrid (Bologna Midline Distractor from KLS Martin laboratory). 
the entire arch. Our laboratory work conducted in the anatomy laboratory at Bordeaux Hospital has allowed us to very objectively compare the tooth-supported and bone-supported distractors. Successive distractions were performed on the same anatomical specimen (as such anatomical conditions are rigorously identical). In terms of the final width of the expansion, the measurements corroborate the similarities between the two types of distractors. ${ }^{27}$
On the other hand, some studies have shown that bone anchorages cause more periodontal and infectious complications, such as irritations of the labial and alveolar mucosa, gingivitis, and infections surrounding the bone fixation system. This is explained by the vestibular position of an actuator and by its anchorage system. For these reasons, Alkan' recommends first using a tooth-supported distractor. A hybrid or bone-supported distractor

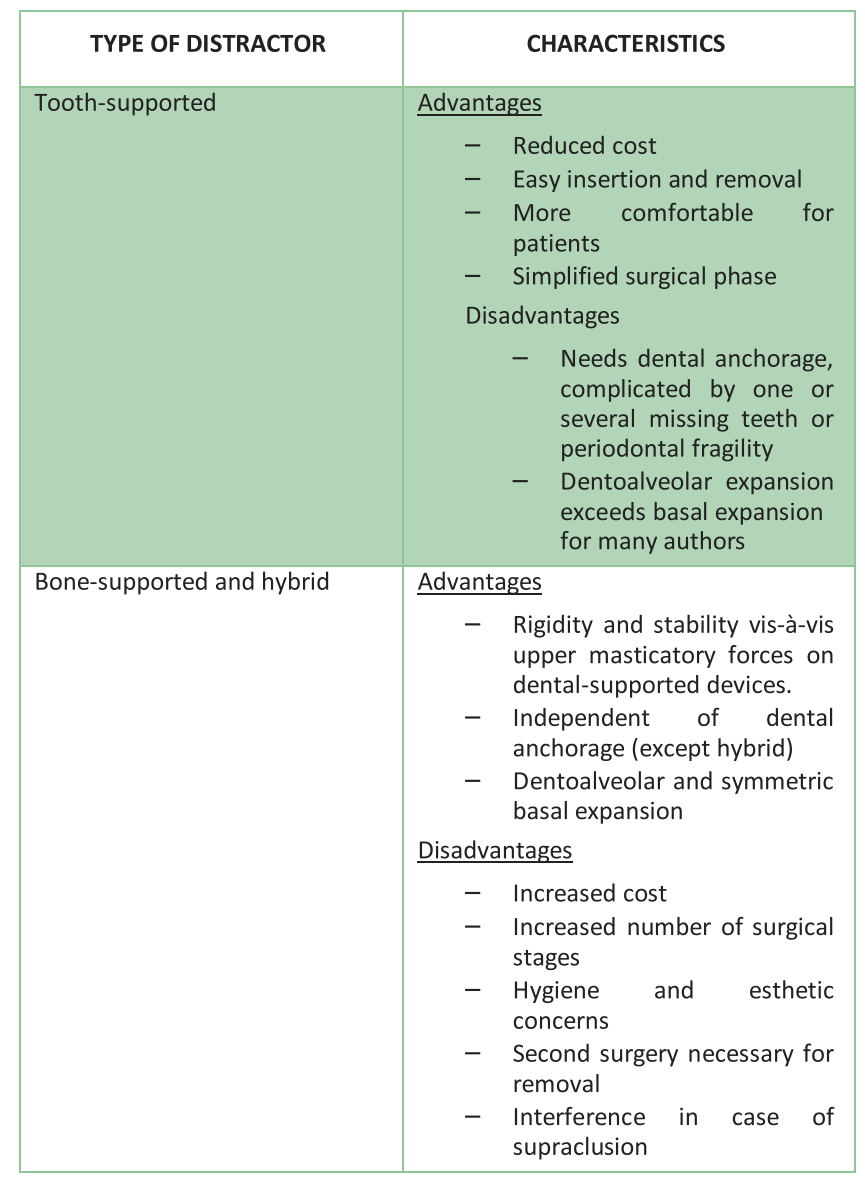

Figure 5

Criteria for choosing different types of distractors. 
is considered in specific edentulous cases and when the anterior zone is too narrow to lingually insert the actuator on the mandibular incisors.

\section{The surgical phase (fig. 6)}

It begins with a small incision (see image 1) so as to minimize the trauma to the chin muscles ${ }^{6}$ and minimize any remote side-effects to the interdental papillae. The symphyseal area is exposed after the detachment of the mucoperiosteal flap (view 2). Preservation of periosteal integrity serves as a general rule for the proper vascularization of the site after closure. It is currently recommended that the osteotomy be made with the aid of Piezotome, which must be finely and precisely selected (view 3). The separation rises to the level of the interdental septum and is completed at the spatula. Bonesupported distractors will be set up while making the osteotomy. To ensure that two vestibular and lingual cortical bones have been selected, it is imperative to ensure that the diastema appears after activating the distractor (view 4). Finally, the actuator is completely enclosed, and the wound is sutured (view 5).

As this is an ambulatory surgery, each patient is released on the evening of the intervention. The prescribed length of sick leave or recovery is 1 week. Regarding eating, foods must be either liquid or mixed for the first week, and then, soft foods are advised for the next month.

\section{The post-surgical phase: the therapeutic schedule (fig. 7)}

Distraction must only begin after a latency period of approximately 7 days, which corresponds to the time lapse necessary for the initiation of bone healing. Furthermore, according to Mommaerts, this resting phase permit the reattachment of Sharpey's fibers in cases where the radicular surface is exposed ${ }^{24}$. Once these 7 days have passed, a premature consolidation may occur.

To determine the distraction rate, most authors have advocated llizarov's work on limb lengthening. An activation that is too rapid may lead to the absence of union or a fibrous union (pseudarthrosis). On the contrary, an activation that is too slow may cause premature consolidation and would therefore not result in the expansion that is initially desired. The norm seems to be approximately $1 \mathrm{~mm}$ per day. However, it must be pointed out that Mommaerts, Landes, and Weil observed no premature consolidation for daily activations of $0.5 \mathrm{~mm}, 0.6 \mathrm{~mm}$, and $0.75 \mathrm{~mm}$, respectively ${ }^{21,25,35}$.

The consolidation phase conditions determine the success of treatment by osteodistraction. The distractor must be kept in place for the entire period, which could last for 2-3 months after the end of active distraction. Given that the initial scar tissue is essentially fibrous and therefore has no mechanical resistance, mobilizing fragments at this stage may cause a fibrous or delayed union, a collapse, or even a fracture ${ }^{2}$. If the mineralization of regenerated tissue has stopped after 4 weeks, it is still premature as it will then undergo a remodeling phase that starts in the sixth week.

After its removal, the distractor should be replaced with a lingual arch 


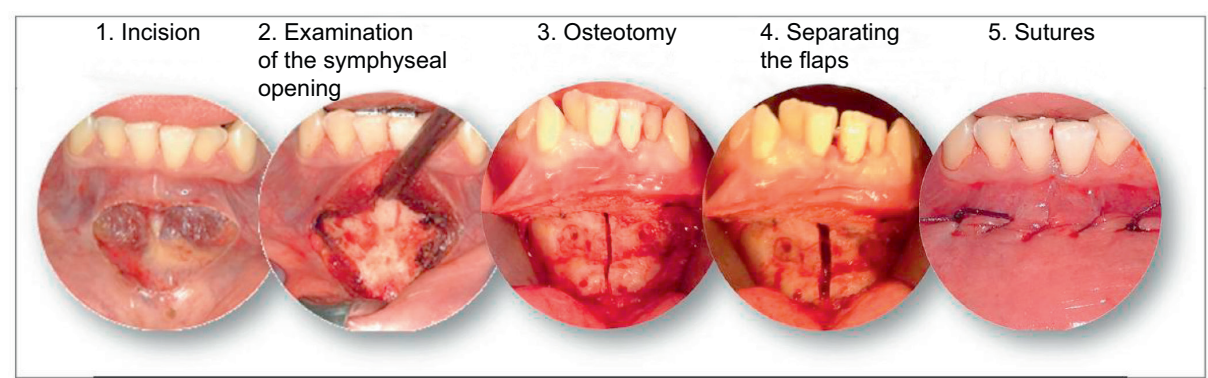

Figure 6

Stages of surgical intervention. Surgery done by Dr. Laurentjoye.

that is well adjusted laterally to obtain desired results. This is much more important at the beginning of orthodontic treatment because the arches used in the first phases of alignment do not allow for the proper maintenance of arch widths. It is a precautionary measure to ensure that the results are maintained. Owing to the follow-up of patients for 18 months, 22 months, and 7 years ${ }^{9,17,20}$, the stability of the expansion obtained from a symphyseal distraction was demonstrated.

\section{Post-distraction: the orthodontic phase}

In current practice, choosing the ideal moment to initiate tooth displacement in the distraction site is a topic of debate. In the first weeks following the intervention, the tissue formed between the bone segments is essentially fibrous. There must be a weak resistance to tooth displacement within this tissue, which explains the phenomenon of "walking tooth" described by some authors ${ }^{5,14,25}$. This results in spontaneous, mesial deviation of teeth within the created space. Some authors mention preventing the premature migration of the incisors while others mention that maxillary displacement can be considered if it is a spontaneous, unprovoked displacement ${ }^{15,17,22}$. It can be achieved at the end of the distraction without impacting the dental validity, radicular integrity, and periodontal health. In a 2015 publication, Garreau ${ }^{12}$ recommended the resumption of orthodontic displacement 15 days after the end of distraction, which would permit (by the phenomena of apposition and reabsorption on the alveolar bone) an acceleration of bone maturation. Of the 35 patients in that study, none had complications linked to early movement. 


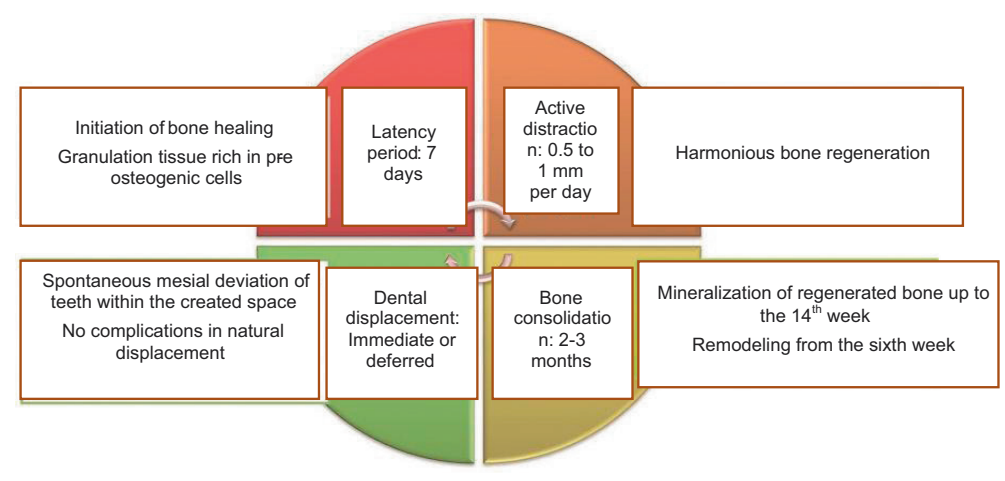

Figure 7

Therapeutic protocol for symphyseal distraction.

\section{NATURE AND MANAGMENT OF COMPLICATIONS}

\section{Complications localized to the surgical area}

In the absence of large-scale clinical studies, the first step has been to isolate all studies that explicitly discussed the occurrence of complications from the literature. Regrouping them has allowed us to reconstitute a unique, homogenous, dataset with a global sample of 277 patients. If the method does not exclude the presence of bias, the resulting chart (fig. 8) shows the frequency of each type of complication identified 1 1,8,12,14,15,18,25,26,34,35.

In light of the data from the literature, mucosal irritation is by far the most frequent complication. It is primarily observed in cases involving the use of bone-supported distractors and hybrids where the actuator is situated in the vestibular area. It is not serious, but it can still influence the decision to switch to a tooth anchorage device.

\section{Periodontal Complications}

Gingival recessions remain quite rare and can be managed by a graft if necessary. This condition seems to become more prevalent with the use of hybrid or bone-supported distractors. More frequently, gingivitis may be observed due to the lack of hygiene in the distractor zone, thus causing a temporary increase in the number of cases. Edema of the gingival sulcus is reversible once a patient resumes a normal brushing routine. Mommaerts ${ }^{25}$ and Kewitt ${ }^{18}$ have measured an average increase in the mobility of the incisor block. Movements are then minimized as ossification progresses. Finally, even if it has not been proven, we can suppose that exposure of the root when making the osteotomy represents a risk of apical migration of epithelial attachment or of ankylosis. As a precautionary measure, a bone of sufficient thickness must be present at 
interradicular sites to preserve the desmodontal ligament and reduce the risk of exposure ${ }^{5,14}$.

\section{Dental Complications}

Radicular fractures are the main risks of the osteotomy. However, of 256 patients, only 3 cases have been reported in the literature. To manage this incident, we can remove the associated fracture fragment by performing root canal or even tooth extraction. A negative response to tests is provisional in some case and is explained by a state of apical contusion. Two cases of loss of pulp vitality have been observed on both sides of the osteotomy; one case of radicular reabsorption has also been observed.

\section{Technical complications}

Technical complications such as an incomplete symphyseal opening, a faulty device mechanism, and premature bone consolidation due to an excessive latency period can cause the distraction to fail. If identified in time, these problems (mishandling a patient or an unstable actuator) can be reversed by performing several activations at once under local anesthesia. If not, a second intervention is required to repeat the osteotomy.

\section{Post-surgical and infectious complications}

Post-surgical and infectious complications such as an abscess or hematoma (following a buccal floor lesion), transient paresthesia, and ptosis of the chin linked to trauma in the mentalis muscle are all within the domain of a surgeon. Regarding these complications, all cases reported in the literature are not very serious infections and are very often located around the anchorage system of bone-supported distractors ${ }^{26}$.

To our knowledge, no study has yet identified a case of pseudarthrosis, which is a complication associated with bone healing. Therefore, it can be deduced that the three types of distractors are strong enough to avoid the onset of pseudarthrosis. Notwithstanding the lack of evidence, it must still be treated as a possible complication of MSDO.

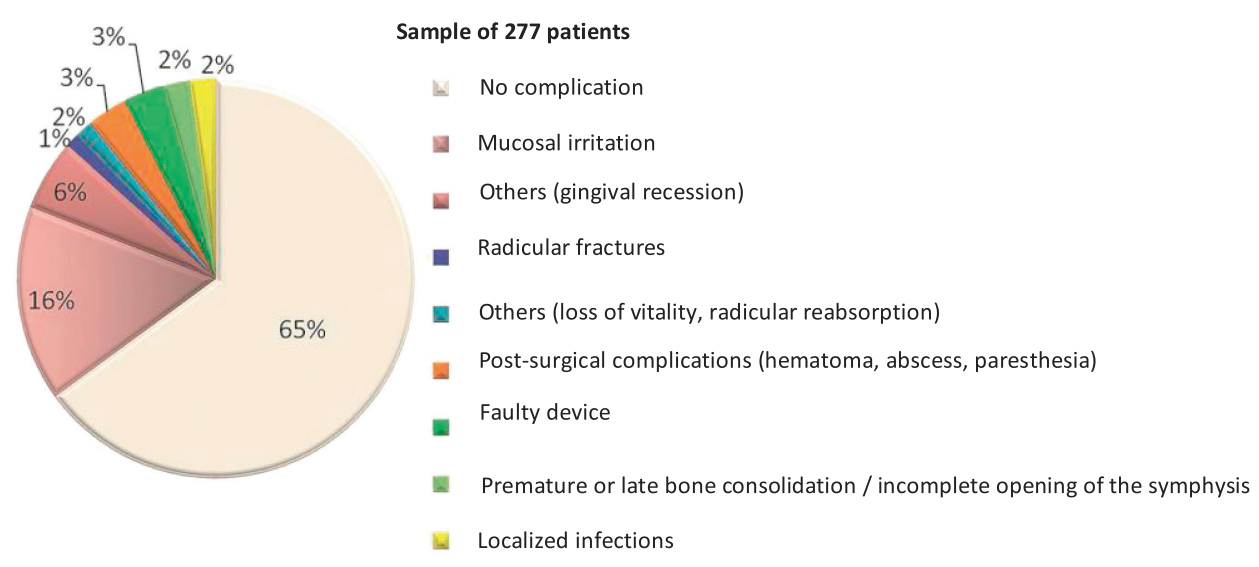

Figure 8

Nature and frequency of complications localized to the zone of intervention. 


\section{Temporomandibular joint disorders}

As the actual effects on temporomandibular joints remain unknown, this partly explains the reticence shown by some practitioners vis-a-vis MSDO. It must be stated that mandibular biomechanics at the time of the distraction as well as the impact on the condyles are difficult to perceive. Routine radiographies are not able to clarify these points.

Irrespective of the type of distractor used, force is applied to the anterior zone of the mandible on the dentoalveolar arch, while the center of rotation is located close to the condyles. Thus, the effects of the distraction are mainly located in the incisor-canine sector and then progressively reduce toward the second molars (fig. 9). The impact becomes minimal at the condyle level, which undergoes slight translational displacement and/or posterolateral rotation.

To evaluate condylar displacements, teleradiographic analysis of the face was used as reference. The analysis has shown that these displacements generally did not exceed $1 \mathrm{~mm}$.

However, the tomodensitometic examination is better to objectively measure the nature and amplitude of these movements considering the limitations of the two-dimensional image in terms of the superimposition of anatomical structures. Our study ${ }^{27}$, mentioned above, allowed us to apply several acquisitions while avoiding the risk of radiation, bearing in mind that the work was conducted on an anatomical specimen.

The measurements obtained using Osirix software have revealed a decrease in the intercondylar distance of $0.93 \mathrm{~mm}$ for the tooth-supported distractor and an increase of $0.17 \mathrm{~mm}$ for the bone-supported distractor. The trends in condylar rotation are more difficult to determine but seem to be weak in amplitude. The conclusions are similar to those outlined in the literature, namely a very minimal impact of distraction at the condylar level, and this result is irrespective of the type of distractor. In total, 14 articles have dealt with the clinical consequences of such displacements $17,712,14,15,18,20,21,26,29,30,35,32,34$. From a sample of 262 patients, only 11 developed transient joint disorders (pains or clacking) that were spontaneously corrected by kinesiology, muscle relaxation splints, or due to a reduction in the speed of the distraction from 1 $\mathrm{mm}$ to $0.5 \mathrm{~mm}$ per day. Six patients experienced regression or even dis-

\section{Expansion average $7 \mathrm{~mm}$}

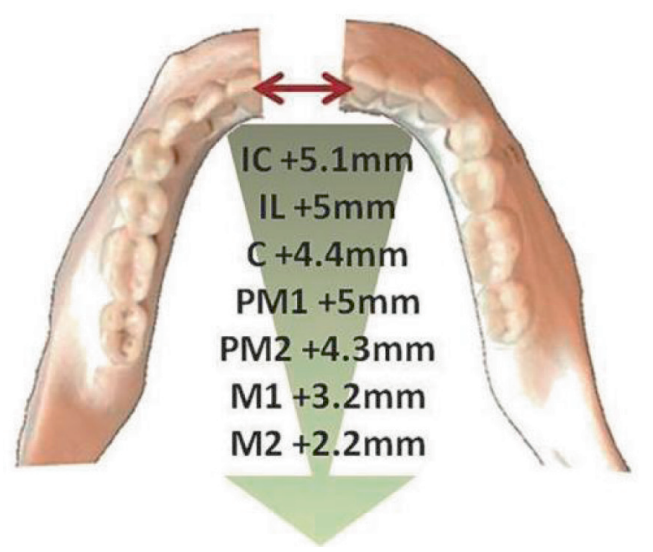

Figure 9

Average number of interdental spaces obtained in different studies: IC (central incisors); IL (lateral incisors); C (canines);

PM1 (first premolars); PM2 (second premolars); $M 1$ (first molars); and M2 (second molars). 
appearance of symptoms after the intervention, owing to the type of care given (fig. 10).

Therefore, neither the high stress level measured by Basciftci's criteria ${ }^{3}$ nor the histological modifications to the superficial condylar layers identified in monkeys by Harper ${ }^{16}$ seem to have major clinical effects on the temporomandibular joints. It would seem that the condyle has a capacity for physiological adaptation, which allows it to adjust itself to the constraints endured. Moreover, Gökalp has suggested that the rate and rhythm of distraction are more important than the total amount of expansion gained ${ }^{13}$. A slower rhythm would permit physiological adaptation and thereby result in functional equilibrium as confirmed by Garreau ${ }^{12}$. The distraction procedure progressively excludes the symphysis, which then permits the adaptation of joints to the rotational trend and/or lateral displacement.

A complete assessment of the temporomandibular joint (TMJ) (inspection, auscultation, and palpation) is strongly recommended in the preoperative phase to detect patients with articular disorders and to anticipate complications. If the condyles seem to adapt to the constraints, which are minimal, encountered during distraction, kineosiological treatment is obligatory after the intervention.

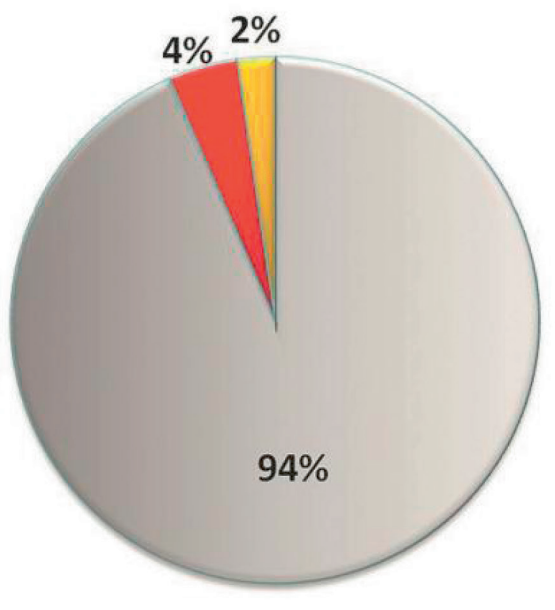

Total sample of 262 patients

No appearance or aggravation of CMD (245 patients)

Transient CMD (11 patients)

Partial or total reduction (6 patients)

Figure 10

Repartition of the articular after-effects observed. 


\section{SOME CLINICAL CASES}

\section{Case no. 1 (figs. 11 and 12)}

This patient is 26 years old and presented with the following:

- Skeletal and occlusal class II associated with a canine-incisal open bite in need of orthodonticsurgical care.

- Maxillary prognathia linked to the transverse compensation trend on the mandibular arch (excessive lingual tilt in the lateral sectors).

- On the mandibular arch, an overlap of $5 \mathrm{~mm}$, premature loss of 36 , and an exact tilt of the inferior incisor into the symphysis.

In this patient, we chose to pair intermaxillary disjunction with symphyseal distraction. In the second stage, mandibular advancement surgery would permit the achievement of skeletal and occlusal class I. Owing to the bimaxillary disjunction, we have been able to re-establish harmonious arch forms and to correct overcrowding (fig. 11D) without having to resort to premolar extractions, which could cause further contraction of the arches. The expansion on the mandible was less significant than that on the maxilla (fig. 11B), but it did not allow for correction of the anterior overlap (5 mm) without allocating the inferior incisor (fig. 12B); orthodontic correction of the lateral torques did not affect the arches.
Note that the distractor was attached to the premolars with composite. The periodontal area of 31 to 41 was unaffected. The visible recession during distraction was only temporary and spontaneously disappeared after closing the diastema (figs. 12F and $G$ ). The septum is shaped like an arrowhead (fig 12D).

\section{Case 2 (figs. 13 and 14)}

This patient is 30 years old and was diagnosed with severe OSAHS (AHI of 25). The patient also had skeletal and occlusal class III and strong maxillary prognathia caused by severe overcrowding of the two arches. A double surgical distraction, the maxilla and mandibular, has been proposed before any treatment in the sagittal direction by bimaxillary advancement. In this patient, considering that dentomaxillary disharmony was severe, symphyseal distraction of 4.5 $\mathrm{mm}$ will not be enough to obtain an alignment of the anterior region, and in part, this is because the actuator's screw thread degraded during activation, which brought about a forced and quick end to the distraction under local anesthesia.

The option to extract the lower incisor, which will have little impact on the perimeter of the arch, was enforced following the symphyseal distraction to manage residual overcrowding (fig. 14C). It should be noted that esthetic makeup was applied to the diastema between 11 and 21 upon the patient's request (fig. 14B). 

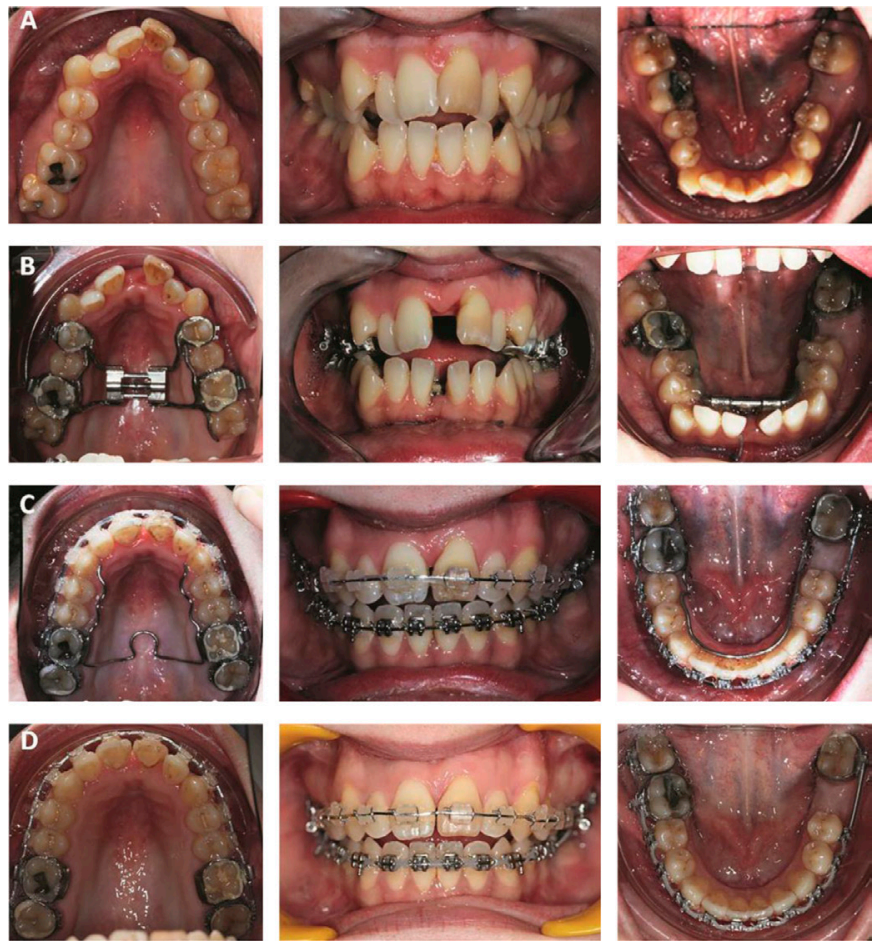

Figure 11

Case 1: Intraoral views before and after bimaxillary distraction. (A) Initial situation. (B) End of distraction. (C) Closure of the spaces and contention of the expansion by the transpalatal and lingual arches. (D) End of orthodontic preparation.

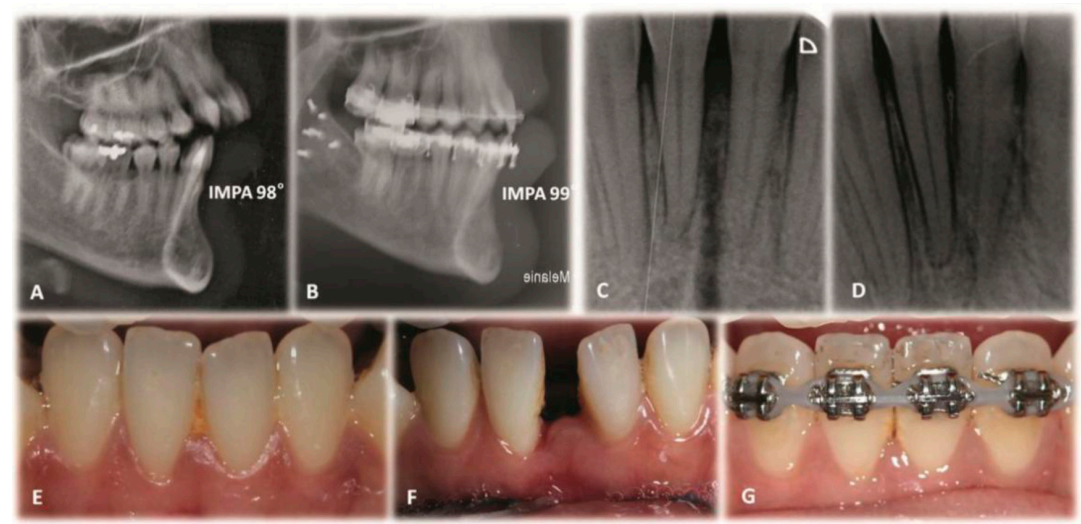

Figure 12

Case 1: Position of the incisors and periodontal area. Position of the incisor in the symphysis (A, B). Retro-alveolar control at 8 months (C) and 20 months (D). Preoperative periodontal environment (E) at the end of the distraction (F) during orthodontic treatment. 

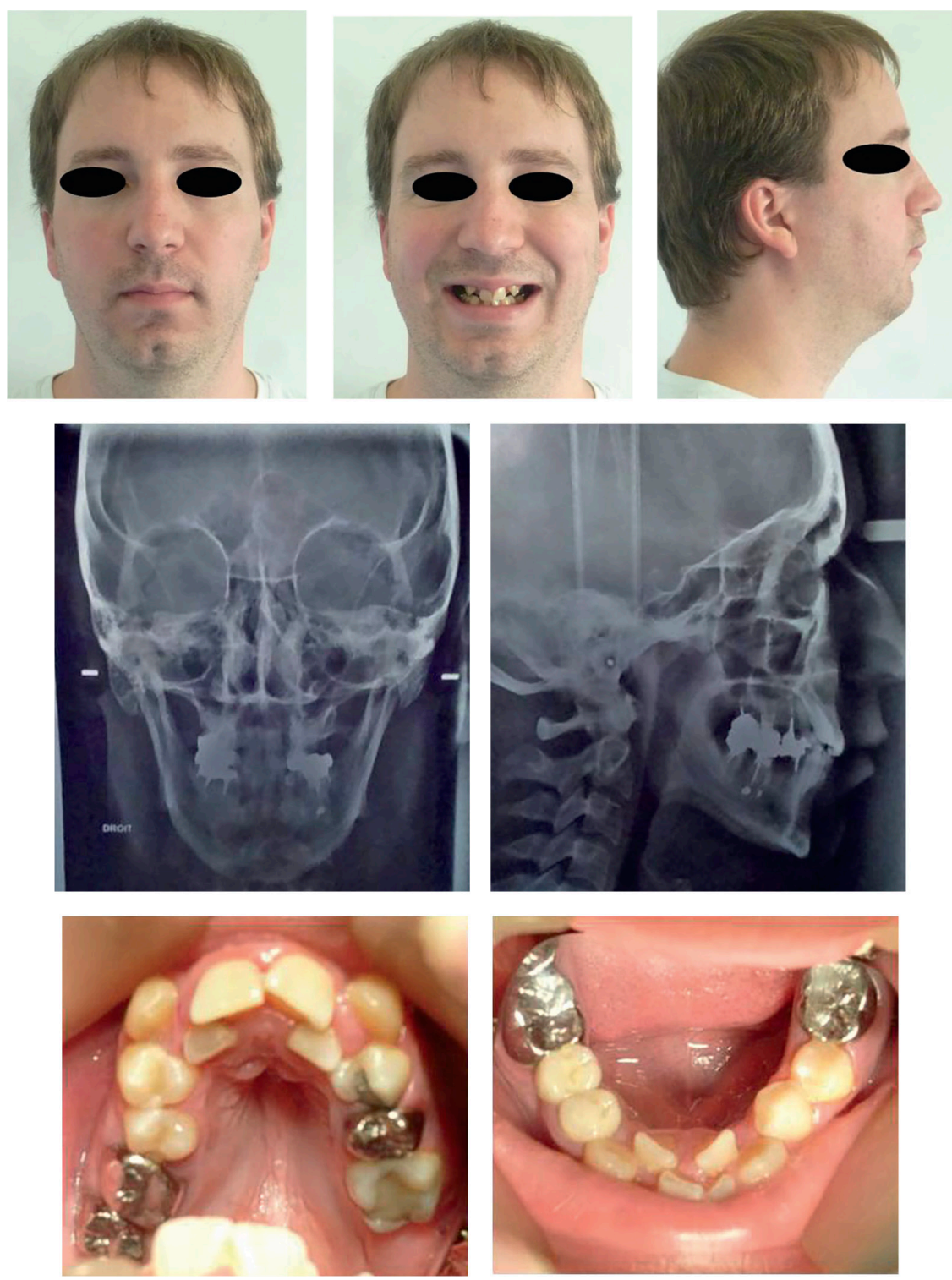

Figure 13

Case 2: The initial situation. 

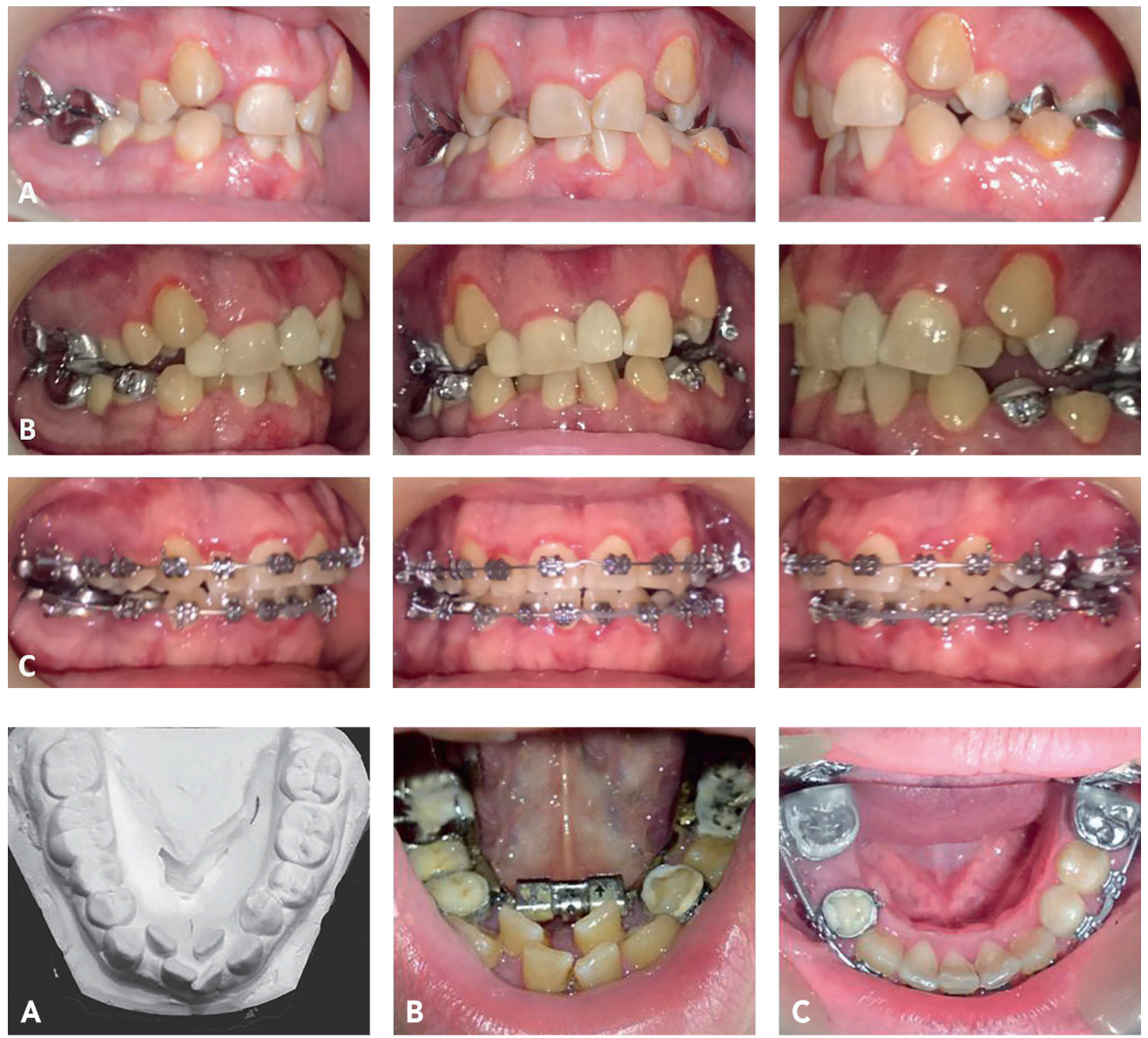

Figure 14

Case 2: (A) Initial intraoral views. (B) At the end of the distraction. (C) During orthodontic preparation.

\section{CONCLUSION}

The data put forward in the literature are intended to provide reassurance regarding the use of symphyseal distraction as it is constantly described as an effective and reliable means for expanding or increasing the mandibular transverse dimension in a durable manner. Although it is more difficult for orthodontists, its implementation and results are similar to those of surgical maxillary disjunction. Most complications tend to be benign and can be anticipated if surgeons and orthodontists collaborate.
More than the actual surgery itself, the difficulty stems from the indications of the symphyseal distraction, which are not always evident. Beyond cases of OSAHS, it should be considered as an alternative to the recourse of extractions when dealing with dentomaxillary disharmony in adolescents or adults and a fortiori when the clinical situation requires a large palatal expansion.

Conflict of interest: The authors declare that they do not have any conflict of interest. 


\section{BIBLIOGRAPHY}

1. Alkan A, et al. Mandibular symphyseal distraction osteogenesis: review of three techniques. Int J Oral Maxillofac Surg 2007;36:111-117.

2. Aronson J. Experimental and clinical experience with distraction osteogenesis. Cleft Palate Craniofacial J 1994;31:473-482.

3. Basciftci FA, Korkmaz HH, Ișeri H, Malkoç S. Biomechanical evaluation of mandibular midline distraction osteogenesis by using the finite element method. Am J Orthod Dentofacial Orthop 2004;125:706-715.

4. Bell WH, Gonzalez M, Samchukov ML, Guerrero CA. Intraoral widening and lengthening of the mandible in baboons by distraction osteogenesis. J Oral Maxillofac Surg 1999;57:548-563.

5. Bell WH, Harper RP, Gonzalez M, Cherkashin AM, Samchukov ML. Distraction osteogenesis to widen the mandible. Br J Oral Maxillofac Surg 1997;35:11-19.

6. Bouletreau P, Paulus C. Correction chirurgicale des anomalies squelettiques transversales maxillo-mandibulaires. Int Orthod 2012;10:261-273.

7. Braun S, Bottrel JA, Legan HL. Condylar displacement related to mandibular symphyseal distraction. Am J Orthod Dentofacial Orthop 2002;121:162-165.

8. Von Bremen J, Schäfer D, Kater W, Ruf S. Complications during mandibular midline distraction. Angle Orthod 2008;78:20-24.

9. Chung Y-W, Tae K-C. Dental stability and radiographic healing patterns after mandibular symphysis widening with distraction osteogenesis. Eur J Orthod 2007;29:256262.

10. Conley RS, Legan HL. Mandibular symphyseal distraction osteogenesis: diagnosis and treatment planning considerations. Angle Orthod 2003;73:3-11.

11. Conley RS, Legan HL. Correction of severe obstructive sleep apnea with bimaxillary transverse distraction osteogenesis and maxillomandibular advancement. Am J Orthod Dentofacial Orthop 2006;129:283-292.

12. Garreau É, Wojcik T, Rakotomalala H, Raoul G, Ferri J. Recours à la distraction symphysaire dans le cadre d'un traitement d'orthodontie: à propose d'une série de 35 cas. Int Orthod 2015;13:81-95.

13. Gökalp H. Effects of symphyseal distraction osteogenesis on the temporomandibular joint seen with magnetic resonance imaging and computerized tomography. Am J Orthod Dentofacial Orthop 2008; 134: 689-699.

14. Guerrero CA, Bell WH, Contasti GI, Rodriguez AM. Mandibular widening by intraoral distraction osteogenesis. Br J Oral Maxillofac Surg 1997;35:383-392.

15. Gunbay T, Akay MC, Aras A, Gomel M. Effects of transmandibular symphyseal distraction on teeth, bone, and temporomandibular joint. J Oral Maxillofac Surg 2009;67:2254-2265.

16. Harper RP, Bell WH, Hinton RJ, Browne R, Cherkashin AM, Samchukov ML. Reactive changes in the temporomandibular joint after mandibular midline osteodistraction. $\mathrm{Br}$ J Oral Maxillofac Surg 1997;35:2025.

17. Ișeri $\mathrm{H}$, Malkoç $\mathrm{S}$. Long-term skeletal effects of mandibular symphyseal distraction osteogenesis. An implant study. Eur J Orthod 2005;27:512-517.

18. Kewitt GF, Van Sickels JE. Long-term effect of mandibular midline distraction osteogenesis on the status of the temporomandibular joint, teeth, periodontal structures, and neurosensory function. J Oral Maxillofac Surg 1999;57:1419-1425.

19. King JW, Wallace JC. Unilateral Brodie bite treated with distraction osteogenesis. Am J Orthod Dentofacial Orthop 2004;125:500-509. 
20. King JW, Wallace JC, Winter DL. Long-term skeletal and dental stability of mandibular symphyseal distraction osteogenesis with a hybrid distractor. Am J Orthod Dentofacial Orthop 2012;141:60-70.

21. Landes CA, Laudemann K, Sader R, Mack M. Prospective changes to condylar position in symphyseal distraction osteogenesis. Oral Surg Oral Med Oral Pathol Oral Radiol Endod 2008;106:163-172.

22. Liou EJ, Figueroa AA, Polley JW. Rapid orthodontic tooth movement into newly distracted bone after man- dibular distraction osteogenesis in a canine model. Am J Orthod Dentofacial Orthop 2000;117:391-398.

23. Malkoç $\mathrm{S}$, Ușü mez $\mathrm{S}$, Ișeri $\mathrm{H}$. Long-term effects of symphyseal distraction and rapid maxillary expansion on pharyngeal airway dimensions, tongue, and hyoid position. Am J Orthod Dentofacial Orthop 2007;132:769-775.

24. Mommaerts MY. Bone anchored intraoral device for transmandibular distraction. $\mathrm{Br} \mathrm{J}$ Oral Maxillofac Surg 2001;39:8-12.

25. Mommaerts MY, Polsbroek R, Santler G, Correia PE, Abeloos JV, Ali N. Anterior transmandibular osteodistraction: clinical and model observations. J Craniomaxillofac Surg 2005;33:318-325.

26. Mommaerts MY, Spaey YJE, Soares Correia PE, Swennen GR. Morbidity related to transmandibular distraction osteogenesis for patients with developmental deformities. J Craniomaxillofac Surg 2008;36:192-197.

27. Pascon L. Analyse biomécanique du comportement mandibulaire lors de la distraction symphysaire avec deux types de distracteurs: étude d'acquisitions tridimensionnelles sur une pièce anatomique. Mémoire. Bordeaux: Université Bordeaux; 2015.

28. Pirelli P, Saponara M, Guilleminault C. Rapid maxillary expansion in children with obstructive sleep apnea syndrome. Sleep 2004;27:761-766.

29. Ploder O, Köhnke R, Klug C, Kolk A, Winsauer H. Three-dimensional measurement of the mandible after mandibular midline distraction using a cemented and screw-fixated tooth-borne appliance: a clinical study. J Oral Maxillofac Surg 2009;67:582-588.

30. Raoul G, Wojcik T, Ferri J. Outcome of mandibular symphyseal distraction osteogenesis with bone-borne devices. J Craniofac Surg 2009;20:488-493.

31. Del Santo M, Guerrero CA, Buschang PH, English JD, Samchukov ML, Bell WH. Longterm skeletal and dental effects of mandibular symphyseal distraction osteogenesis. Am J Orthod Dentofacial Orthop 2000;118:485-493.

32. Seeberger $R$, et al. Changes in the mandibular and dento-alveolar structures by the use of tooth borne mandibular symphyseal distraction devices. J Craniomaxillofac Surg 2011;39:177-181.

33. Sinclair PM, Little RM. Maturation of untreated normal occlusions. Am J Orthod 1983;83:114-123.

34. Uckan S, Guler N, Arman A, Mutlu N. Mandibular midline distraction using a simple device. Oral Surg Oral Med Oral Pathol Oral Radiol Endod 2005;100:85-91.

35. Weil TS, Van Sickels JE, Payne CJ. Distraction osteogenesis for correction of transverse mandibular deficiency: a preliminary report. J Oral Maxillofac Surg 1997;55:953960. 